\title{
Current concepts on the morphology of popliteus tendon and its clinical implications
}

\author{
J. Zabrzyński1,2, G. Huri , A. Yataganbaba ${ }^{3}$, Ł. Paczesny ${ }^{1}$, D. Szwedowski ${ }^{4}$, \\ A. Zabrzyńska ${ }^{5}$, Ł. Łapaj², M. Gagat ${ }^{6}$, M. Wiśniewski ${ }^{7}$, P. Pękala ${ }^{8,9}, 10$ \\ ${ }^{1}$ Department of Orthopaedics, Orvit Clinic, Citomed Healthcare Centre, Torun, Poland \\ 2Department of General Orthopaedics, Musculoskeletal Oncology and Trauma Surgery, \\ University of Medical Sciences, Poznan, Poland \\ ${ }^{3}$ Orthopaedics and Traumatology Department, Hacettepe Universitesi, Ankara, Turkey \\ ${ }^{4}$ Orthopaedic Arthroscopic Surgery International (OASI) Bioresearch Foundation Milan, Italy \\ ${ }^{5}$ Department of Radiology, Multidisciplinary Hospital, Inowroclaw, Poland \\ ${ }^{6}$ Department of Histology and Embryology, Faculty of Medicine, Nicolaus Copernicus University in Torun, \\ Collegium Medicum in Bydgoszcz, Poland \\ ${ }^{7}$ Department of Normal Anatomy, Faculty of Medicine, Nicolaus Copernicus University in Torun, \\ Collegium Medicum in Bydgoszcz, Poland \\ 8International Evidence-Based Anatomy Working Group, Department of Anatomy, Jagiellonian University \\ Medical College, Krakow, Poland \\ ${ }^{9}$ Faculty of Medicine and Health Sciences, Andrzej Frycz Modrzewski Krakow University, Krakow, Poland \\ ${ }^{10}$ Lesser Poland Orthopaedic and Rehabilitation Hospital, Krakow, Poland
}

[Received: 28 July 2020; Accepted: 11 August 2020; Early publication date: 2 September 2020]

In this review we described the anatomy and biomechanics of popliteus muscle and its tendon. Furthermore, we combined the anatomy with clinics and discussed a wide spectrum of disorders regarding the popliteus and its musculotendinous complex. There are three main anatomical regions of the popliteus musculotendinous complex: the proximal origin, the mid-portion, the distal part on the tibia. The unique localisation and various origins of the tendon, connected with structures such as fibular head, Wrisberg, Humphrey and posterior cruciate ligament, lateral meniscus, medial collateral ligament, give an implication to diagnosis and treatment. Popliteus dysfunction is often overlooked, that is the reason why diagnosis and treatment of its injuries is mostly insufficient. Repetitive or acute direct varus forces, when the tibia is in external rotation, and knee hyperextension or flexion with forced external rotation of the tibia, are the main mechanisms of trauma. Popliteus injuries mainly affect the athletic population and lead to severe activity limitations. Chronic disorders of the popliteus tendon, less known, are often described as tendinopathy and are frequently seen in runners. Their symptoms can mimic the lateral meniscal tears. On the other hand, high-energy traumatic injuries of the popliteus tendon often accompany complex, multi ligamentous injuries seen in competitive sports. We also presented the implication of popliteus tendon in knee arthroplasty, due to its particular exposition to iatrogenic trauma during surgery. The issues such as proper tibial component location and well-designed cut systems are crucial to avoid the popliteus impingement and preserve its structure. (Folia Morphol 2021; 80, 3: 505-513)

Key words: popliteus muscle, tendinopathy, posterolateral corner, popliteus reconstruction, popliteus tendon 


\section{ANATOMY}

Popliteus muscle originates from the lateral femoral condyle. It connects with the proximal fibula as a popliteofibular ligament and the posterior horn of the lateral meniscus as a tendinous attachment, and inserts into the posterior surface of tibia above the soleal line [8, 34]. Taylor and Bonney [48] in their comparative study concluded that the popliteus muscle is analogous to the deep portion of the pronator teres muscle. They both originated primitively from the fibula and the ulna, respectively. During the evolution, the origins of these muscles migrated proximally to the lateral femoral condyle and medial humeral epicondyle. Moreover, in reptiles and primitive mammals, the fibula articulates with the femur and subsequently, during the evolution, the fibula has moved distally to the proximal tibiofibular articulation [11].

The popliteus tendon, forming a strong cord, is intra-capsular structure that runs deep to the lateral collateral ligament (LCL), and passes through the popliteal hiatus [37]. Nevertheless, the popliteus tendon lies extra-articular and extra-synovial $[19,34]$. The average total length of the popliteus tendon to its musculotendinous junction is $54.5 \mathrm{~mm}$ [26].

There are three main anatomical regions of the popliteus musculotendinous complex: the proximal origin (A), the mid-portion (B), the distal part on the tibia (C).

A. The proximal origin. The popliteus tendon passes beneath the $\mathrm{LCL}$, and its fibres are attached to the popliteal groove, however, the main fascicle of fibres is inserted underneath the LCL (Fig. 1, 2) [50]. Moreover, it was found that the centre of the femoral insertion of the popliteus muscle-tendon complex is situated posterior and distal to the lateral epicondyle of femur [50].

B. The mid-portion. The popliteus complex has mainly the following origins: the small pit on the lateral femoral condyle, the posterior aspect of the fibrous capsule of the knee joint, the ligamentous band extending between the popliteal tendon and the superior portion of the posterior horn of the lateral meniscus and the popliteofibular ligament [34]. Moreover, the connections with the ligaments of Wrisberg and Humphrey, and the posterior cruciate ligament (PCL) were described [33]. The presence of a fibular attachment of the popliteus, known as popliteofibular (PFL) ligament, was described by numerous authors and is considered as part of the normal anatomy now [8]. Popliteomeniscal fascicles are synovial structures between the lateral meniscus and popliteus tendon. The

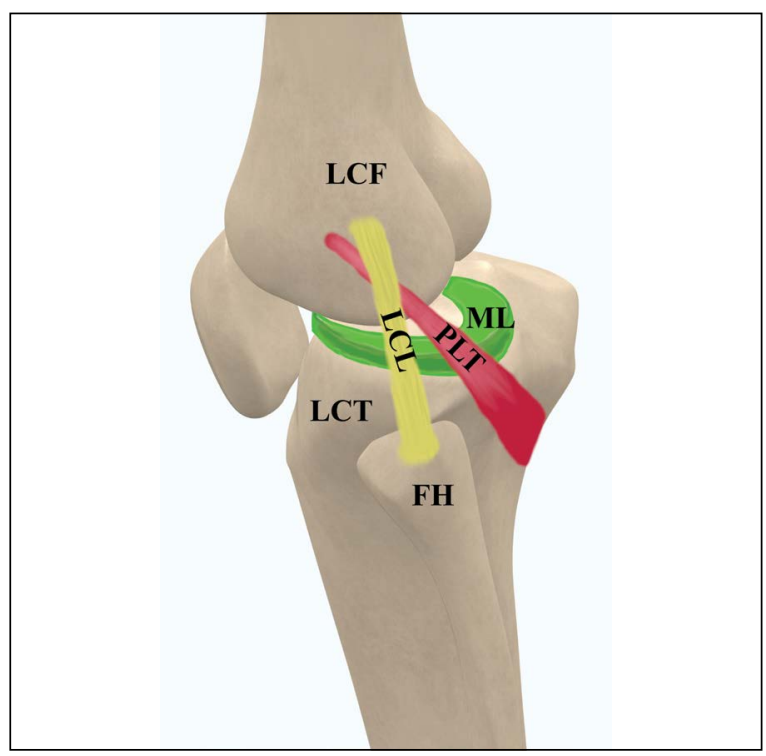

Figure 1. Scheme of the popliteus musculotendinous unit; $\mathrm{FH}$ fibular head; LCF — lateral condyle of the femur; LCL — lateral collateral ligament; $\mathrm{LCT}$ — lateral condyle of the tibia; $\mathrm{ML}$ — lateral meniscus; PLT — popliteus tendon.

anterosuperior, posterosuperior, and posteroinferior popliteomeniscal fascicles, serve as struts, stabilising the posterior horn of the lateral meniscus [2, 37]. Nevertheless, numerous variabilities of the origins were observed [3]. Tria et al. [49], in cadaveric study, reported that $18 / 40$ specimens (45\%) had an isolated popliteus tendon insertion to the lateral femoral condyle, with no connection to the lateral meniscus. However, this results could be biased by the dissection technique or previous morbidities, and on the other hand, Aman et al. [2] revealed the presence of minimum $2 / 3$ of popliteomeniscal fascicles in all examined by them cadaveric limbs. Disruption of the popliteomeniscal fascicles may lead to the abnormal mobility of the lateral meniscus. Simonnet et al. [42] identified the three types of meniscal attachments of the popliteus complex. The authors showed fewer alterations in the lateral meniscus and tibiofemoral cartilage in specimens with more popliteomeniscal fascicles. However, injuries to the popliteomeniscal fascicles are extremely difficult to identify by physical examination and even magnetic resonance imaging (MRI). The MRI is a well-established non-invasive imaging technique in recognition of normal popliteomeniscal fascicles; however, the gold standard for diagnosis and treatment of their tears is arthroscopy [17]. The popliteus tendon is localised in the posterolateral corner of the knee (PLC), in the bony groove, which also has an essential impact on further clinical 

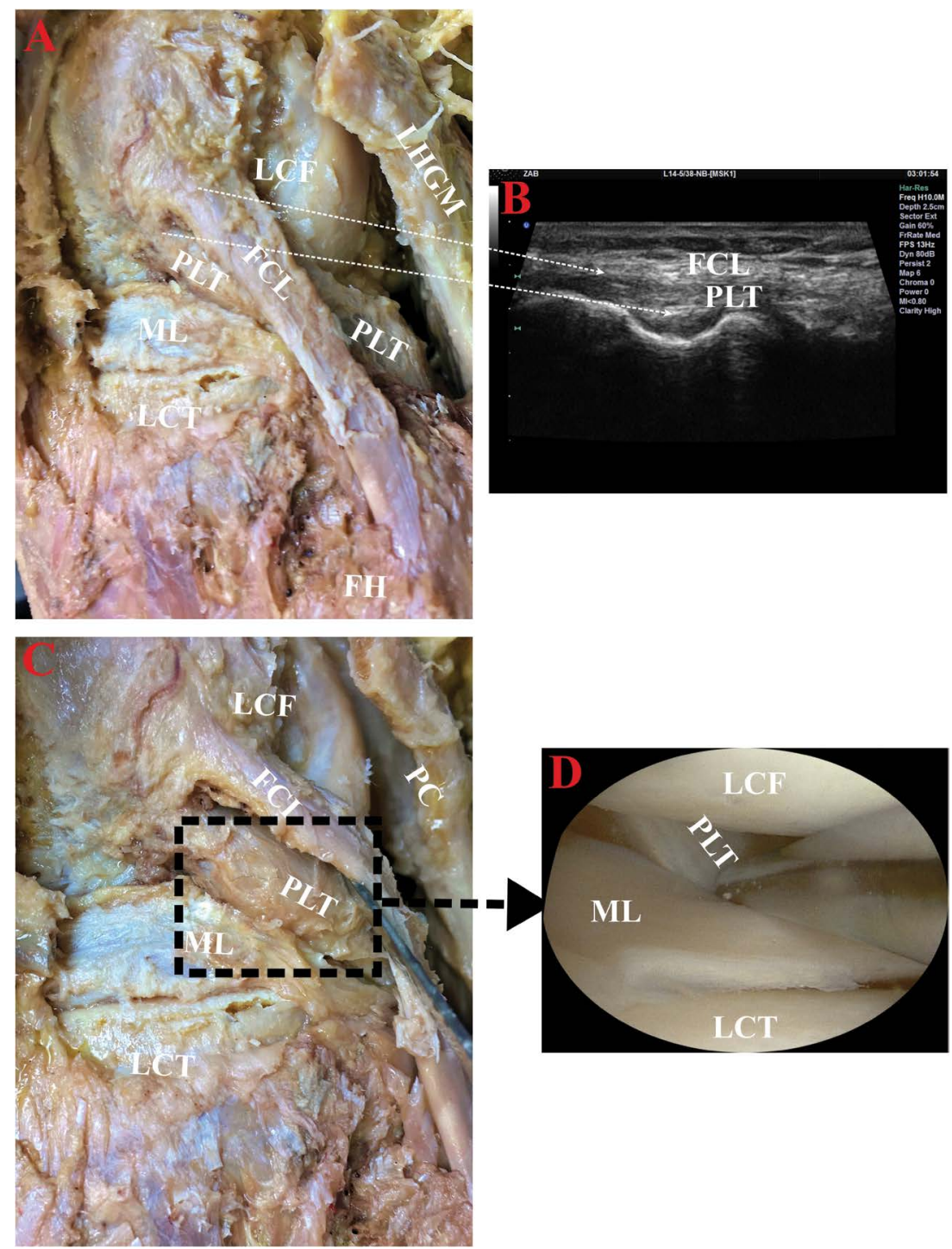

Figure 2. A. Lateral view of the knee joint showing the femoral attachments of popliteus tendon (PLT) and fibular collateral ligament $(\mathrm{FCL})$ to the lateral condyle of the femur (LCF). The PLT passes beneath the FCL, which distal attachment is the fibular head (FH). Moreover, the relation between the lateral meniscus (ML) and the popliteus tendon is visible. Lateral condyle of the tibia (LCT); lateral head of the gastrocnemius muscle (LHGM); B. The sonographic scan of the lateral aspect of the knee joint with the proximal attachments of $\mathrm{FCL}$ and beneath the PLT origin; C. Lateral view of the knee joint showing the PLT and its relation to $\mathrm{ML}$, the $\mathrm{FCL}$ is separated by the probe. Posterior capsule (PC);

D. Arthroscopic view of the lateral compartment of the knee joint and the relations of the PLT to concomitant structures. issues $[11,27]$. The proximal part of the popliteus unit is separated from the lateral collateral ligament, capsule, and lateral femoral condyle by a synovial bursa. If the bursa becomes inflamed, a fluid collection can be seen on MRI or ultrasound imaging [12].

C. The distal part on the tibia. The muscle belly of the popliteus inserts above the soleal line at the proximal and posterior part of the tibia, forming the floor of popliteal fossa $[13,14]$. Song et al. [43] with a three-dimensional reconstruction of the human knee showed that the popliteus tendon is divided into two bundles (medial and lateral) at the popliteal fossa. The popliteus muscle is composed of deep and superficial layers [37]. Some of its distal fibres are interconnected with fascial fibres attached to the distal region of the medial (tibial) collateral ligament (MCL) [33].

\section{BIOMECHANICS}

The popliteus complex acts as static and dynamic stabilizer of the knee joint. Its primary function is to rotate the femur externally when the foot is in contact with the ground and to internally rotate the tibia when the foot is not fixed, which is crucial while walking. During concentric activation, the popliteus internally rotates the tibia; contrary, during eccentric activation, it serves as a secondary restraint to external tibial rotation [33]. This dynamic and static resistance to external rotation is more noticeable, with higher degrees of knee flexion [39].

When the foot is in contact with the ground and the knee is in full extension, the knee is "locked", and initiation of the flexion of the joint requires the popliteus function. It plays a key role that unlocks the knee by rotating the femur externally on the tibia while the 


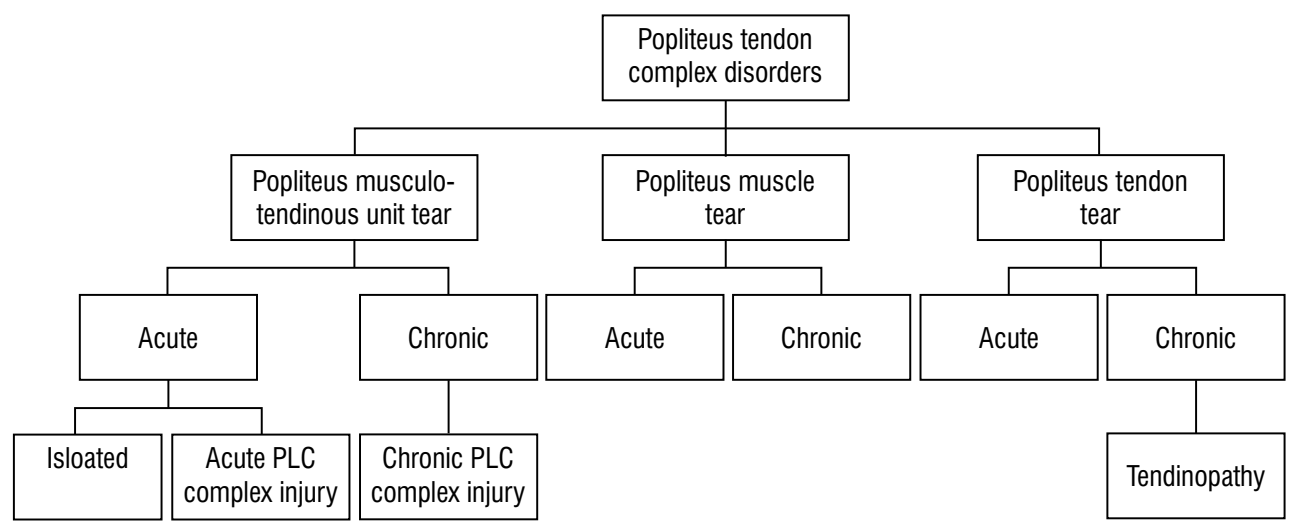

Figure 3. The general classification of the popliteus complex disorders; PLC — posterolateral corner of the knee.

knee is locked, facilitating the initial flexion [19]. Meanwhile, the popliteus' connections to the lateral meniscus and posterior capsule protect the lateral meniscus from impingement during movement. LaPrade et al. [27] defined the popliteus tendon as the "fifth major ligament of the knee". Some studies emphasized that popliteus helps PCL and quadriceps muscle carrying the load, which prevents the femur from excess dislocation. The connection between popliteus and PCL is provided by the Wrisberg and Humphrey ligaments and the main role plays the medial aponeurosis of the popliteus complex $[35,36]$. In a biomechanical in vitro study, it has been shown that when the popliteus tendon is stretched with $50 \mathrm{~N}$, the tibia rotates $4-5$ degrees $\left({ }^{\circ}\right)$ while the knee is fully extended. The amount of rotation increases up to $12^{\circ}$ as the knee is flexed $90^{\circ}$ [23]. Moreover, authors transected the popliteofibular ligament, LCL, popliteus tendon sequentially during cyclic biomechanical testing. They noted gradually increased tibial external rotation with a lateral shift of the position of neutral tibial alignment. During the first 30-degree knee flexion, LCL contributes more to prevent the tibial varus, while the popliteus contributes more to limit the external rotation and posterior translation of the tibia [33].

\section{CLASSIFICATION OF THE PATHOLOGY}

Commonly, the popliteus muscle and tendon disorders are classified into isolated pathology of tendon, muscle belly and complex posterolateral corner injuries of the knee (Fig. 3). Posterolateral corner injuries are divided into three groups: A, B, and C, according to lesions occurring in different structures [13]. Type A involves the PFL and popliteus tendon; in this group, only an increase in the external tibial rotation is observed. In type $\mathrm{B}$, the PFL, popliteus tendon, and LCL are affected. In this group, a lateral gapping occurs under the varus stress test at $30^{\circ}$ of knee flexion along with an increase in tibial external rotation. In type $\mathrm{C}$, the injury to the $\mathrm{PFL}$, popliteus tendon, LCL, lateral capsule (avulsion) and cruciate ligament tear are observed. Severe varus instability at $30^{\circ}$ of knee flexion and in extension is typical. The lack of a comprehensive, prognostic classification system was one of the concepts of the consensus on PLC of the knee presented by Chahla et al. [9]. A future classification system should allow differentiation between structure involved, the type of injury (avulsion versus intrasubstance), the chronicity, the treatment strategy, and it should reflect the prognosis.

\section{MECHANISM OF TRAUMA}

There are various types of specific pathomechanisms that cause popliteus injury. These are: a direct varus force when the tibia is in external rotation and knee hyperextension or flexion with forced external rotation of the tibia [11, 32, 38]. Brown et al. [6] emphasized that the mechanism of trauma is more complex than thought and still has unknown aspects. While the musculotendinous junction is the weakest part of the popliteus complex, the tendon is the most durable structure. The strength of the muscle belly is between these two. The tendon is susceptible to strain at the joint line or avulsion at its origin on the lateral femoral condyle. Moreover, complications related to the popliteus may also occur during total knee arthroplasty. Takubo et al. [47] revealed that the femoral origins of LCL and popliteus tendon are especially exposed to iatrogenic trauma during the knee arthroplasty, due to anatomic conditions. Furthermore, Takakashi 
et al. [45] demonstrated the special design of the cut systems, preserves the popliteus tendon, particularly endangered in a female cohort. The size of the tibial component in the knee arthroplasty also may have an impact on popliteus biomechanics and disorders. Bonnin et al. [5] presented in their cadaveric study, using the computed tomography, the importance of the tibial components location on the tibial plateau and the association with tibio-popliteus impingement. Regarding to knee arthroplasty and stability, Kesman et al. [22] showed that role of the popliteus complex in the stability of the knee joint during the arthroplasty was not clinically important and significant. Contrary, Cottino et al. [10] in their laboratory study showed that popliteus dissection caused both the lateral and medial instability of the knee joint; however, the greater impact was in the lateral compartment.

\section{ISOLATED POPLITEUS TRAUMA AND TENDINOPATHY}

Isolated injuries of the popliteus are rare and usually occur in athletes. Musculotendinous unit lesions are divided into three groups according to the severity of the trauma. Accordingly, grade-1 indicates microtrauma, while grade-3 corresponds to isolated high-energy injuries [30]. Repetitive stress and microtrauma can lead to popliteus tendinopathy. Patients present with persistent and chronic pain in the posterolateral region of the knee, around the popliteus insertion site on the femur, along the tendon and its attachment to the muscle belly $[16,19,38]$. In some cases, symptoms can mimic the lateral meniscal tear. Sometimes, there may be difficulty with walking on uneven ground or going up and down stairs [8]. As stated above, the function of the popliteal unit is to restrain the lateral femoral condyle movements and maintain its relationship with lateral tibial plateau. The downhill running or walking can exacerbate the pathology, causing increased stress on popliteus musculotendinous unit [36]. Patients can run for short distances, but posterolateral knee pain can develop with continued running. In sports such as basketball, tennis, and running, the balance between the femur and the tibia may be impaired due to the development of quadriceps failure. In this case, the load on popliteus increases, and an injury may occur.

\section{COMPLEX POPLITEUS TRAUMA AND PLC INJURY}

On the other hand, high-energy trauma can cause acute haemarthrosis and lateral knee pain. Depend- ing on preserved stability of the joint, the isolated injury of the popliteus muscle-tendon unit should be evaluated. Avulsion of the femoral attachment of the popliteus has also been reported [31, 32]. This form of injury is rare $(<10 \%)$ and is often seen as part of complex knee injuries [19]. The structure of the posterolateral corner was once regarded as the "dark side of the knee" due to the complex and variable anatomy with inconsistent terminology used in the literature to describe the structures in this region [39]. However, in recent years, a significant contribution was made to understand the anatomy and biomechanics of the PLC [8]. Today, it is understood that inadequate diagnosis and treatment of injuries involving the posterolateral corner are associated with poor results and knee instability. Popliteus injury is seen in $60 \%$ to $68 \%$ of patients operated for posterolateral corner instability $[20,27]$. Moreover, complete tears of popliteus unit usually are linked with multi-ligamentous injuries of the knee and subsequently need more advanced surgical procedures [8]. Cruciate ligament rupture ( $\mathrm{ACL}$ or PCL) can mask the presence of PLC instability. Isolated $\mathrm{ACL}$ or $\mathrm{PCL}$ reconstruction without regard to PLC injury may result in graft failure. Posterolateral corner injuries are commonly associated with $A C L$ or $\mathrm{PCL}$ rupture, but also medial compartment bone bruises $[8,15]$. Inadequate reconstructive surgery, omitting the PLC deficiency, can lead to early degenerative changes of the knee joint $[8,43]$. There are three major static stabilizers, known as primary stabilizers of the PLC: the fibular (lateral) collateral ligament, the popliteus tendon and the popliteofibular ligament $[26,39]$. In recent studies, the group of structures forming the PLC were extended and according to these studies, the iliotibial tract, long and short heads of the biceps femoris muscle, mid-third lateral capsular ligament, fabellofibular ligament, also known as gastrocnemiofibular ligament, popliteofibular ligament, lateral meniscotibial ligament and posterior capsule also form the PLC $[20,46]$. LCL and PFL act as static stabilizers against varus stress and external tibial rotation during knee flexion below $30^{\circ}$. Popliteus acts as a dynamic stabilizer against external rotation and posterior translation of the tibia [46]. The relationship between $L C L$ and the insertion of popliteus on the lateral femur condyle has been studied in detail [46]. LCL is usually inserted in the postero-distal slope of the apex of the lateral epicondyle, while the popliteus is inserted to the anterior end of the popliteal sulcus [46]. 


\section{DIAGNOSTICS}

Tests that must be performed in evaluating PLC injuries and simultaneous popliteus injuries are: (1) varus stress test (in full extension: FCL, PLC and cruciate ligament injury; in $20-30^{\circ}$ of knee flexion: $\mathrm{FCL}$ and potentially the secondary stabilizers of the PLC), (2) the dial test (conducted in 30 and $90^{\circ}$ of knee flexion: an increase in the external tibial rotation of more than $10^{\circ}$ in $30^{\circ}$ of flexion compared to the opposite knee suggests PLC injury and an increase in the external tibial rotation of more than $10^{\circ}$ in 30 and $90^{\circ}$ of flexion compared to the opposite knee suggests PLC and PCL injury), (3) reverse pivot shift test (valgus force is applied when the knee is flexed at $90^{\circ}$, and the tibia is forced to external rotation, then the knee is slowly extended, if the subluxated lateral tibial plateau is reduced when the flexion is decreased to $35-40^{\circ}$, the test is positive), (4) the external rotation recurvatum test (the patient is in the supine position, knee joint extended, the great toe is grasped and the leg lifted from the table, while securing the femur to the table by applying gentle pressure to the anterior distal femur, recurvatum is measured by the amount of heel height in $\mathrm{cm}$, test is performed bilaterally to compare) (Fig. 4) $[8,9]$.

If isolated popliteus pathology or complex PLC lesion is suspected after medical interview and physical examination, the proper radiological imaging is necessary. Firstly, standard radiographic imaging should be performed, including anteroposterior (AP), lateral (LAT), and sunrise views of the knee [16]. Stress radiographs are more sensitive for the diagnosis of PLC injuries; however, taking these radiographs can be challenging due to pain in the acute phase of injury.

LaPrade et al. [25] showed that isolated FCL rupture created an average of $2.7 \mathrm{~mm}$ gapping in the lateral joint space in the varus stress radiograph compared to the intact knee, and more than $4 \mathrm{~mm}$ gapping was associated with grade-3 PLC injury. MRI is essential to assess concomitant injuries and to determine the exact location of the injured structures [24]. Standard MRI sequences are often sufficient to evaluate complex knee injuries. Still, PLC structures can be better visualised using a coronal oblique plane view; however, one must be aware that PFL can be missed to the slice thickness. The popliteus musculotendinous complex lesions are detected in $1 \%$ of all knee MRI studies [19]. They may appear on MRI as an avulsion of the femoral attachment, an irregular contour of the tendon at the popliteal hiatus with surrounding high signal intensity changes due to oedema, or as swollen disorganised muscle fibres with high signal intensity changes within the popliteus muscle [19]. On the other hand, the avulsion of the head of the fibula (arcuate sign) may be an important indicator of posterolateral structures tear. This minor fractured bone fragment is often associated with popliteofibular and fabellofibular ligaments attachment rupture. Moreover, it is an important clinical predictor of posterolateral instability and surgical outcomes [17].

\section{ANATOMICAL IMPLICATIONS INTO TREATMENT}

Regarding the extent of pathology, popliteus injuries can be treated conservatively (grade-1-2 tears) or surgically (grade-3 tears and non-response to conservative treatment after three months).

Non-operative treatment is usually recommended for grade-1 and -2 of popliteus injury with good clinical outcomes [8]. Minimal radiographic changes at 8-year follow-up were found after conservative treatment with early mobilisation protocol [21].

Partial-thickness tendon or muscle tears can be treated with open or minimally-invasive debridement [21]. The intraarticular part of the surgery can be done arthroscopically with favourable outcomes [4, 16, 18]. However, in extra-articular cases, open surgery can be necessary depending on the level of stumps retraction. In avulsion injuries, it is necessary to fix the tendon the attachment site using a screw or anchor [7, 29, 31]. Isolated popliteus reconstruction can be performed in PLC injuries with primary external rotation instability pattern. Furthermore, early and aggressive treatment of grade-3 PLC injuries and surgical reconstruction improve long-term outcomes. It prevents persistent instability, varus thrust, chronic pain, and accelerated cartilage damage [39].

Doucet et al. [12] presented a clinical case of a patient with acute calcific tendinopathy of the popliteus tendon. On ultrasound imaging, hypoechoic changes specific to tendinopathy and calcification in the tendon were seen. The patient underwent ultrasound-guided glucocorticoid injection with good clinical results.

Persistent instability and poor functional outcomes were observed in grade-3 PLC injuries that were treated non-operatively [14]. Thus, several PLC surgical reconstruction techniques were described in the literature $[8,20,27]$. Better results are obtained when PLC injuries are repaired or reconstructed in the 

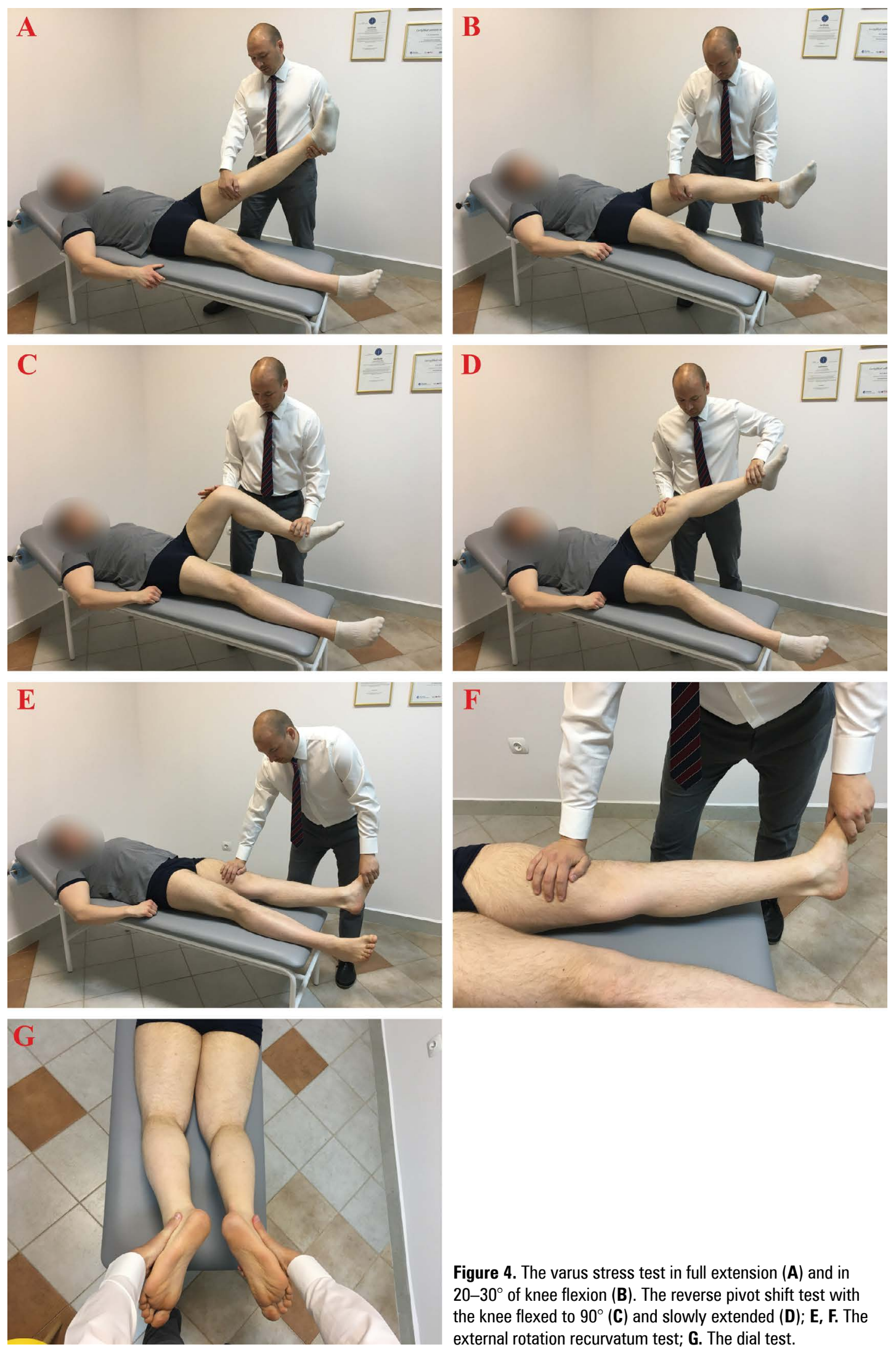

Figure 4. The varus stress test in full extension $(\mathbf{A})$ and in 20-30 of knee flexion (B). The reverse pivot shift test with the knee flexed to $90^{\circ}$ (C) and slowly extended (D); E, F. The external rotation recurvatum test; $\mathbf{G}$. The dial test.

acute stage [41]. Shelbourne et al. [40] reported that repairing the PLC by 4 weeks post-injury resulted in significantly better outcome than a repair performed between 4 to 6 weeks post-injury. 
Non-anatomic reconstruction aims to provide posterolateral stability by applying tension on the uninjured posterolateral structures (extracapsular iliotibial band sling, augmentation technique, bicep tenodesis) [41]. Anatomic reconstruction of the injured LCL, popliteus tendon and PFL is recommended [41]. The reconstructive procedures tend to be more anatomical to restore the knee biomechanics. Stannard et al. [44] and Levy et al. [28] reported lower reoperation rates after PLC reconstruction than PLC repair. Ahn et al. [1] described a novel arthroscopic technique for anatomical reconstruction of the posterolateral corner of the knee joint. This technique reconstructs three key components (LCL, popliteus tendon, PFL) of the posterolateral corner. To obtain better results in the future, surgical techniques that allow early rehabilitation should be developed [8].

\section{SUMMARY}

Popliteus is an essential structure to maintain the knee stability with its sophisticated anatomical and biomechanical features. Its disorders are often overlooked in complex knee injuries. On the other hand, the isolated injuries of popliteus are less frequent, occur mainly in athletes and are characterised by pain around the insertion to the femur, along the tendon and its attachments to the local structures. Thus, in some cases the symptoms can mimic the lateral meniscal tear. Moreover, the imbalance between quadriceps muscle and posterolateral structures can lead to the increase of the load on the popliteus tendon, which can be observed in basketball, tennis, and downhill running. Further studies are needed to improve diagnosis and treatment outcomes, especially in isolated popliteus tendon pathology.

\section{Conflict of interest: None declared}

\section{REFERENCES}

1. Ahn JH, Wang JHo, Lee SY, et al. Arthroscopic-assisted anatomical reconstruction of the posterolateral corner of the knee joint. Knee. 2019; 26(5): 1136-1142, doi: 10.1016/j. knee.2019.07.009, indexed in Pubmed: 31402094.

2. Aman ZS, DePhillipo NN, Storaci HW, et al. Quantitative and qualitative assessment of posterolateral meniscal anatomy: defining the popliteal hiatus, popliteomeniscal fascicles, and the lateral meniscotibial ligament. Am J Sports Med. 2019; 47(8): 1797-1803, doi: 10.1177/0363546519849933, indexed in Pubmed: 31136201.

3. Bartonícek J. Rare bilateral variation of the popliteus muscle: anatomical case report and review of the literature. bartonic@fnkv.cz. Surg Radiol Anat. 2005; 27(4): 347-350, doi: 10.1007/s00276-005-0328-5, indexed in Pubmed: 16228109.
4. Blake SM. Popliteus tendon tenosynovitis. Commentary. Br J Sports Med. 2005; 39(12): e42, doi: 10.1136/ bjsm.2005.019349, indexed in Pubmed: 16306488.

5. Bonnin MP, de Kok A, Verstraete M, et al. Popliteus impingement after TKA may occur with well-sized prostheses. Knee Surg Sports Traumatol Arthrosc. 2017; 25(6): 1720-1730, doi: 10.1007/s00167-016-4330-8, indexed in Pubmed: 27671286.

6. Brown TR, Quinn SF, Wensel JP, et al. Diagnosis of popliteus injuries with MR imaging. Skeletal Radiol. 1995; 24(7): 511-514, doi: 10.1007/BF00202148, indexed in Pubmed: 8545647.

7. Burstein D, Fischer D. Isolated rupture of the popliteus tendon in a professional athlete. Arthroscopy. 1990; 6(3): 238-241, doi: 10.1016/0749-8063(90)90081-n.

8. Chahla J, Moatshe G, Dean CS, et al. Posterolateral corner of the knee: current concepts. Arch Bone Jt Surg. 2016; 4(2): 97-103, indexed in Pubmed: 27200384.

9. Chahla J, Murray IR, Robinson J, et al. Posterolateral corner of the knee: an expert consensus statement on diagnosis, classification, treatment, and rehabilitation. Knee Surg Sports Traumatol Arthrosc. 2019; 27(8): 2520-2529, doi: 10.1007/ s00167-018-5260-4, indexed in Pubmed: 30478468

10. Cottino $U$, Bruzzone $M$, Rosso $F$, et al. The role of the popliteus tendon in total knee arthroplasty: a cadaveric study: SIGASCOT Best Paper Award Finalist 2014. Joints. 2015; 3(1): 15-19, indexed in Pubmed: 26151034.

11. Crum JA, LaPrade RF, Wentorf FA. The anatomy of the posterolateral aspect of the rabbit knee. J Orthop Res. 2003; 21(4): 723-729, doi: 10.1016/S0736-0266(02)00250-4, indexed in Pubmed: 12798074.

12. Doucet C, Gotra A, Reddy SM, et al. Acute calcific tendinopathy of the popliteus tendon: a rare case diagnosed using a multimodality imaging approach and treated conservatively. Skeletal Radiol. 2017; 46(7): 1003-1006, doi: 10.1007/ s00256-017-2623-8, indexed in Pubmed: 28303297.

13. Fanelli GC, Larson RV. Practical management of posterolateral instability of the knee. Arthroscopy. 2002; 18(2 Suppl 1): 1-8, doi: 10.1053/jars.2002.31779, indexed in Pubmed: 11828342.

14. Geeslin AG, LaPrade RF. Location of bone bruises and other osseous injuries associated with acute grade III isolated and combined posterolateral knee injuries. Am J Sports Med. 2010; 38(12): 2502-2508, doi: 10.1177/0363546510376232, indexed in Pubmed: 20837553.

15. Geeslin AG, Moulton SG, LaPrade RF. A Systematic Review of the Outcomes of Posterolateral Corner Knee Injuries, Part 1: Surgical Treatment of Acute Injuries. Am J Sports Med. 2016; 44(5): 1336-1342, doi: 10.1177/0363546515592828, indexed in Pubmed: 26260464.

16. Guha AR, Gorgees KA, Walker DI. Popliteus tendon rupture: a case report and review of the literature. Br J Sports Med. 2003; 37(4): 358-360, doi: 10.1136/bjsm.37.4.358, indexed in Pubmed: 12893726.

17. Huang GS, Yu JS, Munshi M, et al. Avulsion fracture of the head of the fibula (the "arcuate" sign): MR imaging findings predictive of injuries to the posterolateral ligaments and posterior cruciate ligament. AJR Am J Roentgenol. 2003; 180(2): 381-387, doi: 10.2214/ajr.180.2.1800381, indexed in Pubmed: 12540438.

18. Huri G, Biçer OS. Unusual cause of knee locking. Case Rep Orthop. 2013; 2013: 837140, doi: 10.1155/2013/837140, indexed in Pubmed: 23476852.

19. Jadhav SP, More SR, Riascos RF, et al. Comprehensive review of the anatomy, function, and imaging of the popliteus and associated pathologic conditions. Radiographics. 2014; 34(2): 496-513, doi: 10.1148/rg.342125082, indexed in Pubmed: 24617694.

20. Jakobsen BW, Lund B, Christiansen SE, et al. Anatomic reconstruction of the posterolateral corner of the knee: a case series with isolated reconstructions in 27 patients. Arthroscopy. 2010; 26(7): 918-925, doi: 10.1016/j.arthro.2009.11.019, indexed in Pubmed: 20620791. 
21. Kannus P. Nonoperative treatment of grade II and III sprains of the lateral ligament compartment of the knee. Am J Sports Med. 1989; 17(1): 83-88, doi: 10.1177/036354658901700114, indexed in Pubmed: 2929843.

22. Kesman TJ, Kaufman KR, Trousdale RT. Popliteus tendon resection during total knee arthroplasty: an observational report. Clin Orthop Relat Res. 2011; 469(1): 76-81, doi: 10.1007/s11999-010-1525-z, indexed in Pubmed: 20809169.

23. Krudwig WK, Witzel U, Ullrich K. Posterolateral aspect and stability of the knee joint. II. Posterolateral instability and effect of isolated and combined posterolateral reconstruction on knee stability: a biomechanical study. Knee Surg Sports Traumatol Arthrosc. 2002; 10(2): 91-95, doi: 10.1007/ s00167-001-0269-4, indexed in Pubmed: 11914766.

24. LaPrade RF, Gilbert TJ, Bollom TS, et al. The magnetic resonance imaging appearance of individual structures of the posterolateral knee. A prospective study of normal knees and knees with surgically verified grade III injuries. Am J Sports Med. 2000; 28(2): 191-199, doi: 10.1177/03635465000280020901, indexed in Pubmed: 10750995.

25. LaPrade RF, Heikes C, Bakker AJ, et al. The reproducibility and repeatability of varus stress radiographs in the assessment of isolated fibular collateral ligament and grade-III posterolateral knee injuries. An in vitro biomechanical study. J Bone Joint Surg Am. 2008; 90(10): 2069-2076, doi: 10.2106/ JBJS.G.00979, indexed in Pubmed: 18829903.

26. LaPrade R, Ly T, Wentorf F, et al. The Posterolateral Attachments of the Knee. Am J Sports Med. 2017; 31(6): 854-860, doi: 10.1177/03635465030310062101.

27. LaPrade RF, Wozniczka JK, Stellmaker MP, et al. Analysis of the static function of the popliteus tendon and evaluation of an anatomic reconstruction: the "fifth ligament" of the knee. Am J Sports Med. 2010; 38(3): 543-549, doi: 10.1177/0363546509349493, indexed in Pubmed: 20042547.

28. Levy BA, Dajani KA, Morgan JA, et al. Repair versus reconstruction of the fibular collateral ligament and posterolateral corner in the multiligament-injured knee. Am J Sports Med. 2010; 38(4): 804-809, doi: 10.1177/0363546509352459, indexed in Pubmed: 20118498.

29. Liu JN, Rebolledo BJ, Warren RF, et al. Surgical management of isolated popliteus tendon injuries in paediatric patients. Knee Surg Sports Traumatol Arthrosc. 2016; 24(3): 788-791, doi: 10.1007/s00167-016-4029-x, indexed in Pubmed: 26856317.

30. De Maeseneer M, Shahabpour M, Vanderdood K, et al. Posterolateral supporting structures of the knee: findings on anatomic dissection, anatomic slices and MR images. Eur Radiol. 2001; 11(11): 2170-2177, doi: 10.1007/s003300100983, indexed in Pubmed: 11702156.

31. Mirkopulos N, Myer TJ. Isolated avulsion of the popliteus tendon. A case report. Am J Sports Med. 1991; 19(4): 417-419, doi: 10.1177/036354659101900419, indexed in Pubmed: 1897662.

32. Nakhostine $M$, Perko $M$, Cross $M$. Isolated avulsion of the popliteus tendon. J Bone Joint Surg Br. 1995; 77(2): 242-244, indexed in Pubmed: 7706338.

33. Nyland J, Lachman N, Kocabey $Y$, et al. Anatomy, function, and rehabilitation of the popliteus musculotendinous complex. J Orthop Sports Phys Ther. 2005; 35(3): 165-179, doi: 10.2519/jospt.2005.35.3.165, indexed in Pubmed: 15839310.

34. Paraskevas G, Papaziogas B, Kitsoulis P. A study on the morphology of the popliteus muscle and arcuate popliteal ligament. Folia Morphol. 2006; 65(4): 381-384, indexed in Pubmed: 17171619.

35. Peduto AJ, Nguyen A, Trudell DJ, et al. Popliteomeniscal fascicles: anatomic considerations using MR arthrography in cadavers. Am J Roentgenol. 2008; 190(2): 442-448, doi: 10.2214/AJR.07.2643, indexed in Pubmed: 18212231.

36. Petsche TS, Selesnick FH. Popliteus tendinitis: tips for diagnosis and management. Phys Sportsmed. 2002; 30(8): 27-31, doi: 10.3810/psm.2002.08.401, indexed in Pubmed: 20086537.

37. Porrino J, Sharp JW, Ashimolowo T, et al. An update and comprehensive review of the posterolateral corner of the knee. Radiol Clin North Am. 2018; 56(6): 935-951, doi: 10.1016/j.rcl.2018.06.006, indexed in Pubmed: 30322491.

38. Quinlan JF, Webb S, McDonald K, et al. Isolated popliteus rupture at the musculo-tendinous junction. J Knee Surg. 2011; 24(2): 137-140, doi: 10.1055/s-0031-1275397, indexed in Pubmed: 21874950.

39. Rosas HG. Unraveling the posterolateral corner of the knee. Radiographics. 2016; 36(6): 1776-1791, doi: 10.1148/ rg.2016160027, indexed in Pubmed: 27726747.

40. Shelbourne KD, Haro MS, Gray T. Knee dislocation with lateral side injury: results of an en masse surgical repair technique of the lateral side. Am J Sports Med. 2007; 35(7): 1105-1116, doi: 10.1177/0363546507299444, indexed in Pubmed: 17379923.

41. Shon OJ, Park JW, Kim BJ. Current concepts of posterolateral corner injuries of the knee. Knee Surg Relat Res. 2017; 29(4): 256-268, doi: 10.5792/ksrr.16.029, indexed in Pubmed: 29172386

42. Simonnet ML, Rooze M, Feipel V. The proximal attachments of the popliteus muscle: a quantitative study and clinical significance. Surg Radiol Anat. 2003; 25(1): 58-63, doi: 10.1007/s00276-002-0093-7, indexed in Pubmed: 12677465.

43. Song $Y$, Xiong $Y$, Chen $W$, et al. Sectional anatomy and three-dimensional visualization of the posterolateral complex of the knee joint based on undeformed high-resolution sectional anatomical images. Anat Rec (Hoboken). 2018; 301(10): 1764-1773, doi: 10.1002/ar.23926, indexed in Pubmed: 30383338

44. Stannard JP, Brown SL, Farris RC, et al. The posterolateral corner of the knee: repair versus reconstruction. Am J Sports Med. 2005; 33(6): 881-888, doi: 10.1177/0363546504271208, indexed in Pubmed: 15827360.

45. Takahashi A, Sugita T, Aizawa T, et al. Potential risk of excising the femoral insertion of the popliteus tendon during primary total knee arthroplasty: a biometric study. J Orthop Sci. 2015; 20(6): 1030-1035, doi: 10.1007/s00776-015-0773-x, indexed in Pubmed: 26362655.

46. Takeda S, Tajima G, Fujino K, et al. Morphology of the femoral insertion of the lateral collateral ligament and popliteus tendon. Knee Surg Sports Traumatol Arthrosc. 2015; 23(10): 3049-3054, doi: 10.1007/s00167-014-3059-5, indexed in Pubmed: 24839040.

47. Takubo A, Ryu K, Iriuchishima T, et al. The evaluation of the distance between the popliteus tendon and the lateral collateral ligament footprint and the implant in Total knee Arthroplasty using a 3-dimensional template. BMC Musculoskelet Disord. 2020; 21(1): 322, doi: 10.1186/s12891-02003347-6, indexed in Pubmed: 32443975.

48. Taylor G, Bonney V. On the homology and morphology of the popliteus muscle: a contribution to comparative myology. J Anat Physiol. 1905; 40(Pt 1): 34-50, indexed in Pubmed: 17232661.

49. Tria AJ, Johnson CD, Zawadsky JP. The popliteus tendon. J Bone Joint Surg Am. 1989; 71(5): 714-716, indexed in Pubmed: 2732260.

50. Yang JH, Lim HC, Bae JiH, et al. Anatomic and isometric points on femoral attachment site of popliteus muscle-tendon complex for the posterolateral corner reconstruction. Knee Surg Sports Traumatol Arthrosc. 2011; 19(10): 1669-1674, doi: 10.1007/s00167-011-1442-z, indexed in Pubmed: 21328069. 